\title{
Editorial:
}

\section{Emerging concepts in acute respiratory distress syndrome: implications for clinicians}

The acute respiratory distress syndrome (ARDS) was first described by Ashbaugh and colleagues in $1967 .{ }^{1}$ Simply stated, it is a non-cardiogenic pulmonary oedema leading to decreased lung compliance and hypoxia. The Berlin definition classifying $\mathrm{ARDS}^{2}$ has helped to bring clarity to the definition based on severity of ARDS. Further, better understanding of the pathophysiology, has led to changing strategies in the approach and management of ARDS.

Several new concepts regarding the aetiology of ARDS have emerged in the last few years. The first is the acknowledgement of the role of genetics in the predisposition to development of ARDS. ${ }^{3}$ Sepsis is the most important cause of ARDS all over the world, but it is now known that not all patients with sepsis develop ARDS, pointing to unknown host factors. It has been found that epigenetics and gene expression have a major role in the predisposition to ARDS. Measurement of various metabolites in exhaled breath and in bronchoalveolar lavage (BAL) fluid ${ }^{3}$ have been found to be predisposing to ARDS. In future, epigenetic targets may be identified for prevention of ARDS.

The understanding of pathophysiology of inflammation of alveolo-capillary membrane in ARDS has been redefined in the recent times. The neutrophilic infiltration and their activation leading to alveolar oedema is now known to be reaction to the inflammation rather than the cause. This has been proven by the fact that even neutropenic patients develop ARDS and the administration of granulocyte colony stimulating factor (G-CSF) to ARDS patients does not exaggerate ARDS. The role of angiotensin converting enzyme pathways are known to contribute to the fibrotic and proliferative phase of ARDS. ${ }^{4}$

The various phases of inflammation, proliferation and fibrosis in ARDS have been categorized in the last decade. However ventilatory strategies aimed at different phases of ARDS are still being worked out. With the advent of computed tomography (CT), the infiltrates of ARDS are being clearly defined as generally being posterior and basal, pointing to the heterogeneity of the lung in ARDS. There are areas of normal lung, as well as completely inflamed lung. Ventilation of the normal lung with increased pressures aimed at distending the inflamed lung may lead to over distention and barotrauma.

The concept of the so called "baby lung" evolved over the years to indicate that the normally aerated lung in ARDS probably has the dimensions of the lung of a 5 or 6 year old child. ${ }^{5}$ This diminished capacity of the lung in ARDS was thought to be due to the stiffness and decreased compliance of the involved lung. Such involvement was thought to be permanent and anatomically fixed. However, the CT images of patients with ARDS disproved this. When the patient was put in prone position, the infiltrates moved to the anterior part of the chest showing that the posterior oedematous lung is capable of re-expanding and is not permanently stiff. ${ }^{6}$ This has given more

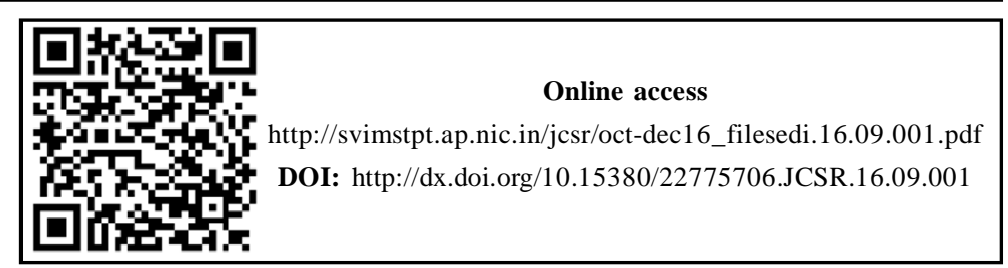


validity for the use of prone-position ventilation as the water-logged lung is allowed to expand and recovery of alveoli is possible.

The next major change in the ventilation strategy in ARDS came with the understanding that ventilation can produce lung injury, the so called "ventilator induced lung injury (VILI)". This concept was described by Tierney et $\mathrm{al}^{8}$ in rats in 2003. Now it is well known that VILI is due to volutrauma which may be caused by over distension of alveoli. This has been the reason for using low tidal volume ventilation of $6 \mathrm{~mL} / \mathrm{kg}$ as opposed to $10 \mathrm{~mL} / \mathrm{kg}$ which was used in earlier years. ${ }^{9}$

Two more types of alveolar trauma have been identified. The first is atelect-trauma due to repetitive opening and closing of alveoli. The application of positive end-expiratory pressure (PEEP) prevents the gross stress and strain on the alveoli, which itself is known to trigger further inflammation. The second measure which allows the driving pressure from the ventilator to be evenly distributed through the inhomogeneous lung is prone position ventilation, thus saving the "baby lung" from excessive distention. Prone positioning removes the weight of the inflammed lung on the posterior alveoli. Both these measures have been shown to be successful in reducing atelect-trauma. ${ }^{10}$

Bio-trauma is due to cytokine release from the damaged alveoli. The concept of biotrauma was discovered after it was noted that most patients with ARDS die from multiple organ dysfunction syndrome (MODS) rather than hypoxia. ${ }^{11}$ This shows that the ARDS afflicted lung may be the source of release of cytokines causing MODS. Several studies have demonstrated the increasing concentrations of tumour necrosis factor-alpha (TNF- $\alpha$ ), interleukin-1 beta (IL-1 $\beta$ ) and macrophage inflammatory protein (MIP-2) in BAL fluid especially with increasing volumes during ventilation. This has further strengthened the concept of low tidal volume ventilation and avoiding repeated recruitment of the lung, as a measure to prevent cytokine storm and possibility of MODS.

Probably the most important change in ventilation strategy with the realization of VILI, is the acceptance of lesser arterial oxygen tension $\left(\mathrm{PaO}_{2}\right)$ as targets during ventilation. Arterial oxygen saturation measured by pulse oximetry $\left(\mathrm{SpO}_{2}\right)$ value of $85 \%-90 \%$ is enough for survival of patients and haemodynamic stability during the course of ARDS. This concept has led to "gentle lung treatment" and acceptance of lesser oxygenation and to some extent permissive hypercapnia. So understanding of pathophysiology has led to lowering of $\mathrm{PaO}_{2}$ targets during treatment of ARDS, and low tidal volume, with less distention of alveoli has been implemented. ${ }^{12}$ Hopefully, this may have major implications in the lowering of mortality in this condition.

The resolution of ARDS involves the reversal of capillary permeability, the establishment of the integrity of the endothelial membrane and stabilization of the cytoskeleton of the alveolus. Several factors and pathways are involved in this process of reversal. Mesenchymal stem cells which may secrete anti-inflammatory cytokines and growth factors may be useful for therapy in hastening recovery. ${ }^{13}$

ARDS has been associated with $40 \%-50 \%$ mortality in several centres across the world. This has been thought of as due to the severity of the disease itself. However, recent studies have shown that polymorphism of the ACE gene may have a role in the prediction of mortality in Asian populations. ${ }^{14}$ After adjusting for several factors, this polymorphism has been shown to definitely affect mortality.

Data about ARDS from developing nations is heavily compromised by the absence of facilities for ruling out cardiogenic pulmonary oedema. In several third world countries, even arterial blood gas assessment is problematic. Keeping this in mind, the Kigali Modifications for the Berlin definition 
has been proposed. The ratio of $\mathrm{PaO}_{2}$ to fraction of inspired oxygen $\left(\mathrm{FIO}_{2}\right)$ can be safely replaced by $\mathrm{SpO}_{2} / \mathrm{FIO}_{2}$ ratio of less than 315 for the diagnosis and assessment of ARDS. Such modifications may lead to more data from third world countries on this devastating condition. ${ }^{15}$

The concepts regarding the etiology of ARDS have shifted focus from environmental factors to host factors. The ventilator strategies have been relooked at with better understanding of the pathophysiology of ARDS after the advent of CT. The recognition of the trauma caused by ventilation has changed the modes of ventilation, the settings for ventilation and the $\mathrm{PaO}_{2}$ and arterial carbon dioxide tension $\left(\mathrm{PaCO}_{2}\right)$ targets. With these concepts, it is hoped that the future will lead to lesser morbidity and mortality for patients with ARDS.

\section{Sudha Vidyasagar Department of Medicine, Kasturba Medical College, Manipal e-mail:vsagar33@yahoo.com}

Received: September 02, 2016; Accepted: September 22, 2016.

Vidyasagar S. Emerging concepts in acute respiratory distress syndrome: implications for clinicians. J Clin Sci Res 2016;5:202-4. DOI: http://dx.doi.org/10.15380/22775706.JCSR.16.09.001.

\section{REFERENCES}

1. Ashbaugh DG, Bigelow DB, Petty TL, Levine BE. Acute respiratory distress in adults. Lancet. 1967;290:319-23.

2. Ranieri VM, Rubenfeld GD, Thompson BT, Ferguson ND, Caldwell E, et al. ARDS Definition Task Force. Acute respiratory distress syndrome: the Berlin Definition. JAMA 2012;307:2526-33.

3. Rogers AJ, Matthay MA. Applying metabolomics to uncover novel biology in ARDS. Am J Physiol Lung Cell Mol Physiol 2014;306:L957-61.

4. Lambert DW, Clarke NE, Turner AJ. Not just angiotensinases: new roles for the angiotensin-converting enzymes. Cell Mol Life Sci 2010;67:89-98.

5. Gattinoni L, Pesenti A. The concept of “baby lung”. Intensive Care Med. 2005;31:776-84.

6. Grasso S, Stripoli T, Sacchi M, Trerotoli P, Staffieri F, Franchini D, et al. Inhomogeneity of lung parenchyma during the open lung strategy: a computed tomography scan study. Am J Respir Crit Care Med 2009;180:415-23.

7. Slutsky AS, Ranieri VM. Ventilator-induced lung injury. N Engl J Med 2013;369:2126-36.

8. Tierney DF. Ventilator-induced lung injury occurs in rats, but does it occur in humans? Am J Respir Crit Care Med 2003;168;1414-5.

9. The Acute Respiratory Distress Syndrome Network. Ventilation with lower tidal volumes as compound with traditional tidal volumes for acute lung injury and the acute respiratory distress syndrome. N Engl J Med 2000;342:1301-8.

10 Gattinoni L, Quintel M. How ARDS should be treated. Critical Care 2016;20:86.

11. Saddy F, Sutherasan Y, Rocco PR, Pelosi P. Ventilator-associated lung injury during assisted mechanical ventilation. Semin Respir Crit Care 2014;35:409-17.

12. Change in goal of ARDS Pesenti A. Target blood gases during ARDS ventilatory management. Intensive Care Med 1990;16:349-51.

13. Matthay MA, Ware LB, Zimmerman GA. The acute respiratory distress syndrome. clin Inv. 2012;122:2731-40.

14. Matsuda A, Kishi T, Jacob A, Aziz M, Wang P. Association between insertion/deletion polymorphism in angiotensin-converting enzyme gene and acute lung injury/acute respiratory distress syndrome: a meta-analysis. BMC Med Genet 2012;13:76.

15. Bein T. From Berlin to Kigali: the sobering journey of acute respiratory distress syndrome. J Thoracic Dis 2016;8:E282-4. 